\title{
Equalizadores Cegos
}

\author{
Roberto S. Lucatelli Araújo e Fábio Violaro
}

\begin{abstract}
Os equalizadores adaptativos convencionais empregados em canal telefônico operam com base em algoritmos de minimização do erro quadrático médio. Esses algoritmos exigem um perfodo de treinamento inicial no quat é transmitida uma sequeência previamente determinada e connecida no receptor. Este trabalho apresenta algoritmos alternativos que dispensam a transmissăo de seqüências especlficas. São descritos os vários algoritmos propostos na literatura até o presente e são mostrados os resultados de algumas simulações realizadas com canais telefornicos.
\end{abstract}

\section{Introdução}

A transmissão de dados em canais com faixa limitada está sujeita a vários tipos de distorções que dificultam a detecção correta do sinal no extremo receptor. Em particular, a interferência intersimbólica (iis), causada pela dispersão do sinal no tempo, é a principal responsável pela degradação do sinal recebido. Com o objetivo de minimizar a iis, o receptor dispõe de um dispositivo denominado equalizador adaptativo, que comumente tem seus coeficientes ("taps") ajustados pelo algoritmo do gradiente estocástico [1]. Como será justificado na Secão 2, este algoritmo necessita de uma seqüência de treinamento para alcançar a convergência inicial, e só após esse treinamento é que ele passa a operar com a seqüência de dados propriamente dita. No entanto, há situações onde seria interessante que o receptor dispen. sasse o recebimento de uma seqüência previamente conhecida para atingir a convergência. Um caso típico, descrito em[2] , ocorre numa rede multiponto já em operação normal, quando um novo terminal deseja acessar a estação central. Claramente, se esta tiver que interromper o fluxo de dados para enviar uma seqüência específica para cada novo terminal chamador, a eficiência da rede cairá bastante. O objetivo deste trabalho é apresentar e discutir algoritmos que sejam capazes de convergir independentemente da seqüênciß recebida e sem conhecê-la a priori. Equalizadores que operam baseados nestes algoritmos são conhecidos como equalizadores cegos ou equalizadores auto-adaptativos.

R. S. L. Araújo é Pesquisador do CPqD - TELEBRÁS, Caixa Postal 1.579, 13.100, Campinas - SP.

F. Violaro \& Professor da Faculdade de Engenharia Elétrica da UNICAMP, Caixa Postal 6.101, 13.081, Campinas - SP. 
Na Seção 2 o algoritmo do gradiente é revisado e é estabelecido o modelo do sistema de transmissão que será utilizado posteriomente. A Seção 3 apresenta os algoritmos de equalização cega propostos na literatura até o momento e a Seção 4 mostra o resultado de algumas simulações realizadas em canais telefônicos típicos.

\section{Algoritmo do Gradiente Estocástico}

A Fig. 1 mostra um sistema de transmissão de dados típico [3]. A seqüência binária $d(n)$ de entrada é codificada numa seqüência complexa $a(n)=a_{r}(n)+$ jai $(n)$, formatada pelo filtro $p(t)$, e modula em quadratura uma portadora de freqüência $f_{c}$. $O$ canal telefônico de resposta impulsional $z(t)$ é de faixa limitada $(300$ a $3.400 \mathrm{~Hz})$ e portanto o sinal de saida $r(t) \dot{e}$, em geral, fortemente afetado pela iis. Além disso, há adicionalmente a degradação causada pelo ruído de canal $n_{c}(t)$. O filtro de recepção, com resposta impulsional $g(t)$, limita o ruído à faixa do sinal e o filtro transversal, com resposta de freqüência $E(f)$, se encarrega de minimizar a iis de forma que a seqüência decidida $\{\hat{a}(n)\}$ seja, de acordo com algum critério, a mais próxima possivel da seqüência transmitida $\{a(n)\}$.

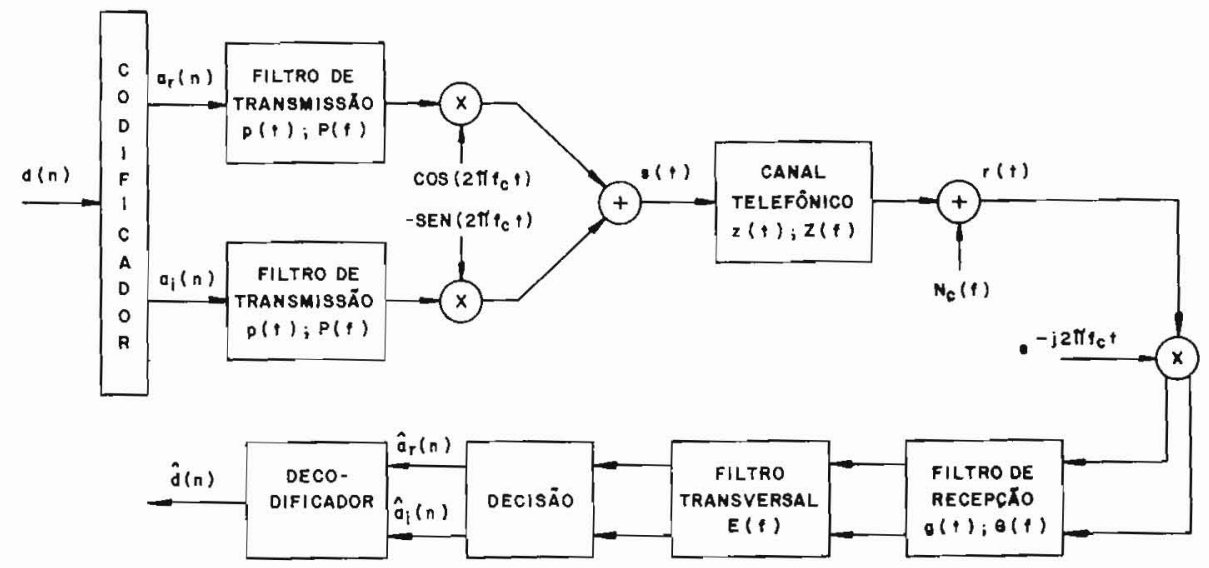

Figura 1. Sistema de transmissão QAM.

A análise do sistema da Fig. 1 é bastante facilitada se os processos de modulação e demodulação forem combinados com os filtros de transmissão, recepção e com o canal de resposta impulsional $z(t)$, dando origem ao sistema equivalente em banda básica [3], mostrado na Fig. 2. O equalizador com resposta de freqüência $E(f)$ é um filtro transversal com resposta impulsional $c(n)$, determinada pelo algoritmo de controle. Este, por sua vez, é alimentado pelo sinal de erro $e(n)$ definido por

$$
e(n)=y(n)-\hat{a}(n)
$$


onde

$$
y(n)=\sum_{j=-N}^{N} c(n) u(n-j)
$$

com

$$
u(n)=\sum_{j} a(j) h(n-j)+n(n)
$$

Em (3), $h(n)$ é a resposta impulsional complexa do canal equivalente passabaixas e $n(n)$ é um ruído gaussiano complexo com amostras independentes. $\mathrm{O}$ algoritmo de controle atua de modo a minimizar o erro quadrático médio (MSE) dado por

$$
M S E=E\left\{|e(n)|^{2}\right\}
$$

onde $E\{$.$\} denota o valor esperado da quantidade entre colchetes.$

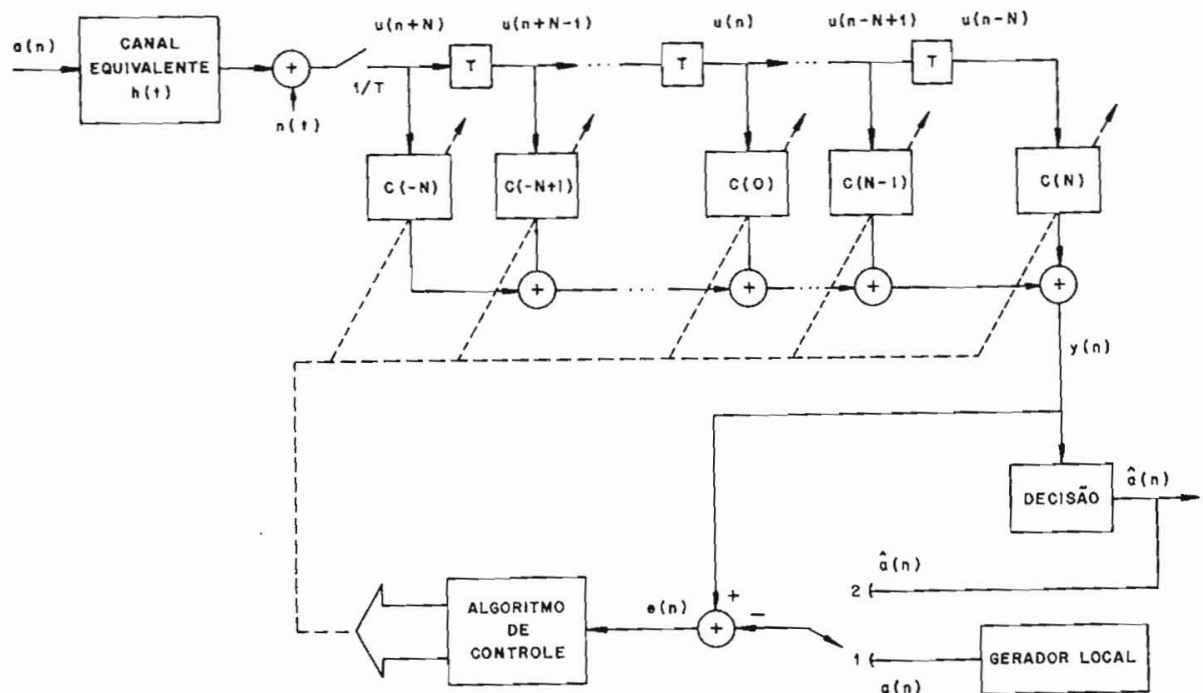

Figura 2. Sistema equivalente em banda básica e equalizador transversal.

Pode-se mostrar [3] que a minimização acima leva ao chamado algoritmo do gradiente estocástico, proposto em 1960 por B. Widrow[4]. Neste algoritmo tem-se

$$
\mathbf{c}(n+1)=\mathbf{c}(n)-\mu \mathbf{u}^{*}(n) e(n)
$$

onde $\mathbf{c}(n)$ é o vetor de coeficientes e $\mathbf{u}(n)$ é o vetor de entrada. Os transportos destes vetores se escrevem, respectivamente, 


$$
c^{\prime}(n)=[c(-N), \ldots, c(0), \ldots, c(N)]
$$

e

$$
u^{\prime}(n)=[u(n+N), \ldots, u(n), \ldots, u(n-N)]
$$

Em (5), $\mu$ é a constante de adaptação e $\mathbf{u}^{\star}(n)$ denota o conjugado do vetor $\mathbf{u}(n)$. Para valores adequados de $\mu$ o algoritmo converge para a solução ótima que minimiza o valor de MSE em (4), desde que as decisões â(n) sejam corretas. Na prática isto não ocorre, isto é, a probabilidade de que, no início do processo, â(n) esteja muito próximo de a(n) é, em geral, muito baixa, ou seja, a taxa de erro é elevada, gerando um sinal de erro $\mathrm{e}(\mathrm{n})$ inadequado à operação do algoritmo em (5). Para contornar este problema é necessário que haja um período de treinamento, no qual uma seqüência previamente determinada seja transmitida. No receptor, um gerador local fornece esta mesma seqüência, em sincronismo com o transmissor, garantindo um sinal de erro e(n) confiável. Portanto, no início da comunicação, a chave que aparece na Fig. 2 está na posição 1. Terminado o treinamento, ela passa para a posição 2 e o equalizador passa a operar com a seqüência de dados propriamente dita.

\section{Equalizadores Cegos}

Como foi dito na seção anterior, o algoritmo definido por (5) exige uma seqüência de treinamento por causa da não-disponibilidade de uma referência confiável no início da comunicação. Pode-se entretanto eliminar o treinamento, definindo-se funções de erro mais robustas que permitam a convergência do algoritmo, independentemente da taxa de erro inicial. A seguir, são apresentadas as diversas funções de erro propostas até o momento na literatura. Deve-se observar que, mesmo nos artigos onde cada uma delas é sugerida, não há uma dedução formal destas funções de erro e nem de que maneira elas foram encontradas. Intuitivamente, pode-se dizer que a idéia central dos algoritmos de equalização cega é a substituição do sinal decidido â(n) por um outro parâmetro que leva em conta valores médios relacionados à constelação de símbolos.

Algoritmo I - Y. Sato 1975 [5]

A função de erro é dada por

$$
e^{s}(n)=y(n)-\beta \operatorname{sgn}[y(n)]
$$

onde

$$
\begin{aligned}
& \beta=E\left\{a_{r}^{2}(n)\right\} / E\left\{\left|a_{i}(n)\right|\right\}=E\left\{a_{i}^{2}(n)\right\} / E\left\{\left|a_{i}(n)\right|\right\} \\
& y(n)=y_{r}(n)+j y_{i}(n)
\end{aligned}
$$

e

$$
\operatorname{sgn}[y(n)]=\operatorname{sgn}\left[y_{r}(n)\right]+j\left[\operatorname{sgn} y_{i}(n)\right]
$$


com

$$
\operatorname{sgn}(x)=\left\{\begin{array}{l}
1 \text { para } x \geqslant 0 \\
-1 \text { para } x \leqslant 0
\end{array}\right.
$$

Algoritmo II - A. Benveniste 1984 [6]

Neste algoritmo, a função de erro se escreve

$$
e^{b}(n)=k_{1} e(n)+k_{2}|e(n)|^{2} e^{s}(n)
$$

onde $k_{1}$ e $k_{2}$ são constantes e e(n) e $e^{S}(n)$ são definidos em (1) e (6), respectivamente.

Algoritmo III - D. Godard 1980 [2]

Tem-se aqui

$$
e^{g}(n)=y(n)\left\{|y(n)|^{2}-R\right\}
$$

onde

$$
R=E\left\{|a(n)|^{4}\right\} / E\left\{|a(n)|^{2}\right\}
$$

Algoritmo IV - K. Wesolowski 1987 [ 7]

A função de erro é agora dada por

$$
e^{w}(n)=y_{r}(n)\left[y^{2} r(n)-A\right]+j y_{j}(n)\left[y^{2}(n)-A\right]
$$

onde

$$
A=E\left\{a_{r}{ }^{4}(n)\right\} / E\left\{a^{2} r(n)\right\}=E\left\{a^{4} i(n)\right\} / E\left\{a^{2}{ }_{i}(n)\right\}
$$

Algoritmo V-G. Picchi 1987 [ 8]

Para este algoritmo, a função de erro é dada por (1),

$$
e^{P}(n)=f_{r}(n) e_{r}(n)+j f_{i}(n) e_{i}(n)
$$

onde $e_{r}(n)$ e $e_{j}(n)$ são as partes real e imaginária de $e(n)$, dado por (1)

$$
f_{r}(n)=\left\{\begin{array}{l}
1, \text { se } \operatorname{sgn}\left[e_{r}(n)\right]=\operatorname{sgn}\left[e_{r}^{s_{r}}(n)\right] \\
0, \text { em caso contrário }
\end{array}\right.
$$




$$
f_{i}(n)=\left\{\begin{array}{l}
1, \text { se } \operatorname{sgn}\left[e_{i}(n)\right]=\operatorname{sgn}\left[e^{s_{i}}(n)\right] \\
0, \text { em caso contrário }
\end{array}\right.
$$

com $e^{S_{r}(n)}$ e $e^{-s_{i}(n)}$ denotando as partes real e imaginária de $e^{S}(n)$, dado por (6).

Várias observações podem ser feitas em conexão com os algoritmos acima definidos.

1) O algoritmo II é uma extensão do algoritmo I e apresenta a vantagem de ter erro tendendo a zero no estado estacionário; isto é, após a convergência, a flutuação dos coeficientes do equalizador em torno do valor ótimo é menor no algoritmo II que no algoritmo I. Isto também ocorre com o algoritmo $\mathrm{V}$, enquanto os algoritmos I, III e IV apresentam flutuações no estado estacionário.

2) A função minimizada pelo algoritmo III é dada por [2]

$$
E\left\{\left[|y(n)|^{2}-R\right]^{2}\right\}
$$

Conseqüentemente, ela não depende da fase do sinal recebido $y(n)$ e, dessa forma, os algoritmos de equalização e recuperação de portadora estão totalmente desacoplados. Além disso, como é mostrado em [2], a convergência se dá independentemente da constelação de símbolos, o que não ocorre com os algoritmos I e II, que apresentam problemas, por exemplo, para a constelação V29 do CCITT [6].

3) O algoritmo IV é uma modificação do algoritmo III e, como será mostrado nas simulações, converge com maior velocidade.

4) Se $f_{r}(n)=1$ e $f_{i}(n)=1$, a função de erro do algoritmo $V$ se reduz àquela dada por (4). Entretanto, as funções $f_{r}(n)$ e $f_{i}(n)$ atuam de forma a levar em conta, com uma probabilidade elevada, somente as decisŏes corretas, e em caso contrário as correções não são efetuadas (daí o nome "Stop-and-Go Decision-Directed Algorithm").

5) A complexidade computacional adicional introduzida pelos algoritmos de equalização cega em relação ao algoritmo convencional é indicada naTabela 1. Observe que o número de operações por iteração não depende do número de coeficientes do equalizador, de modo que o esforço computacional necessário à implementação dos algoritmos cegos é pouco maior que aquele requerido pelo algoritmo convencional. 


\begin{tabular}{l|c|c|c|c|c|c}
\hline \multirow{2}{*}{$\begin{array}{c}\text { Tipo } \\
\text { de } \\
\text { Operação }\end{array}$} & \multicolumn{5}{|c}{ Algoritmo } \\
\cline { 2 - 7 } & Convencional & I & II & III & IV & V \\
\hline sgn (x) & 0 & 2 & 2 & 0 & 0 & 6 \\
multiplicações & 0 & 2 & 14 & 6 & 4 & 4 \\
somas ou subtrações & 2 & 2 & 4 & 3 & 2 & 4 \\
\hline
\end{tabular}

Tabela 1. Número de operações no cálculo da função de erro.

A análise da convergência dos algoritmos descritos não é simples devido às não-linearidades das funções $|x|, \operatorname{sgn}(x), f_{r}(n)$ e $f_{j}(n)$, e ainda não se dispõe na literatura de um resultado concreto a respeito da questão. $A$ única evidência de convergência para casos reais (canal com distorções e ruído) é obtida através de simulações. Algumas tentativas de exame da convergência podem ser encontradas em [9] e [10].

\section{Simulações}

Para avaliar e comparar o desempenho dos algoritmos descritos, considere - sistema da Fig. 1. As respostas em freqüência (amplitude e atraso de grupo) dos dois canais utilizados nas simulações são mostradas nas figuras 3 e 4. Estes canais podem ser considerados típicos e apresentam diferentes razões $\lambda_{\max } / \lambda_{\min }$, onde $\lambda_{\max }$ e $\lambda_{\min }$ correspondem aos autovalores máximo e mínimo da matriz de autocorrelação do sinal à entrada do equalizador. A razão $\lambda_{\max } / \lambda_{\min }$ é uma medida da distorção de amplitude causada pelo canal na seqüência de entrada que é formada por símbolos descorrelatados entre si e que, portanto, possui espectro plano. Para o canal 1 esta razão vale' 34,2 e corresponde a um canal com acentuada distorção de amplitude; para o canal 2 esta razão vale 5,5 e corresponde a um canal com distorção de amplitude moderada. O sistema opera a 9.600 bits por segundo com uma taxa de transmissão de símbolos de 2.400 baud. Os filtros de transmissão e recepção são de fase linear e características de amplitude iguais à raiz quadrada do cosseno levantado com excesso de faixa de $10 \%$. O equalizador transversal possui 31 coeficientes complexos e a constelação de símbolos é aquela mostrada na Fig. 5. A razão sinal-ruído é de $27 \mathrm{~dB}$, medida na entrada do equalizador. Com estes dados pode-se calcular o MSE mínimo a partir de (4), resultando $-21,6 \mathrm{~dB} e-25,85 \mathrm{~dB}$ para os canais $1 \mathrm{e}$ 2 , respectivamente.

Os resultados das simulações são apresentados nas figuras 6 e 7 enquanto na Tabela 2 podem ser encontrados os parâmetros utilizados na implemen- 


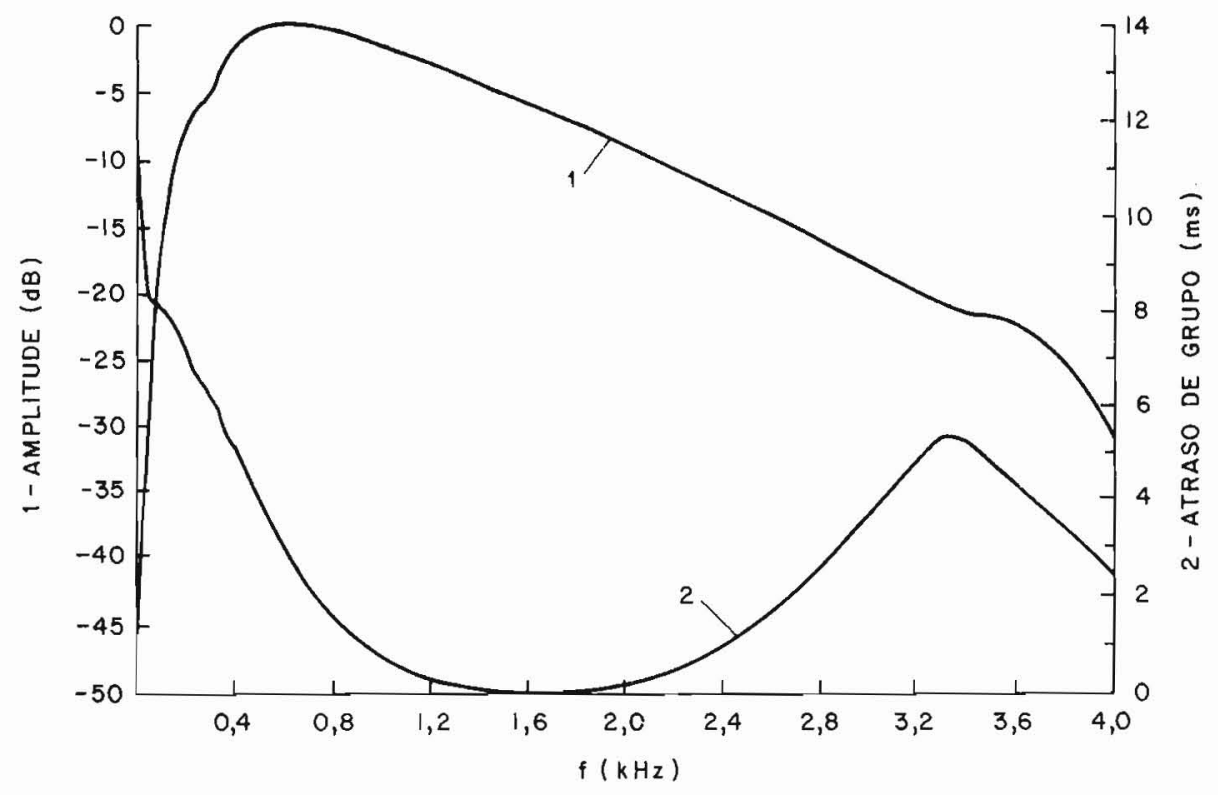

Figura 3. Características de amplitude e atraso de grupo do canal 1 $\left(\lambda_{\max } / \lambda_{\min }=34,2\right)$.

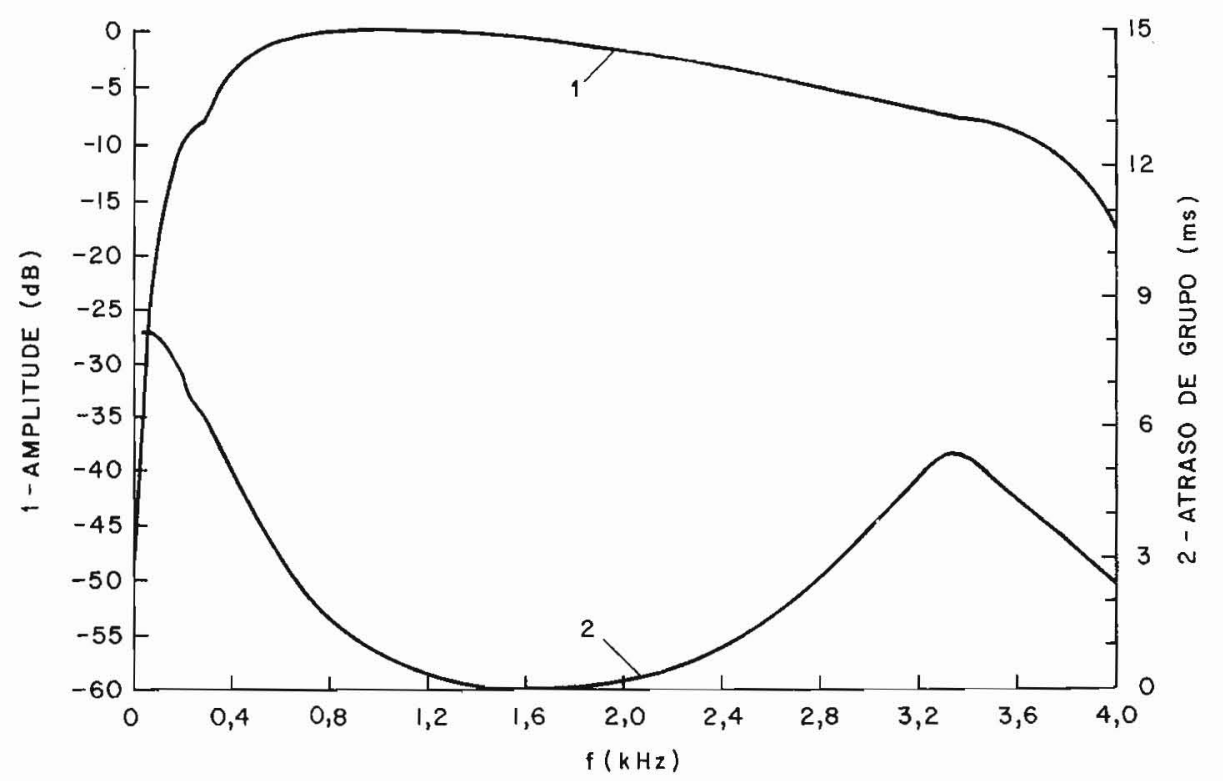

Figura 4. Características de amplitude e atraso de grupo do canal 2 $\left(\lambda_{\max } / \lambda_{\min }=5,5\right)$. 
tação de cada algoritmo. As curvas representam a evolução do erro quadrático médio (MSE), medido em $\mathrm{dB}$, em função do número de iterações.

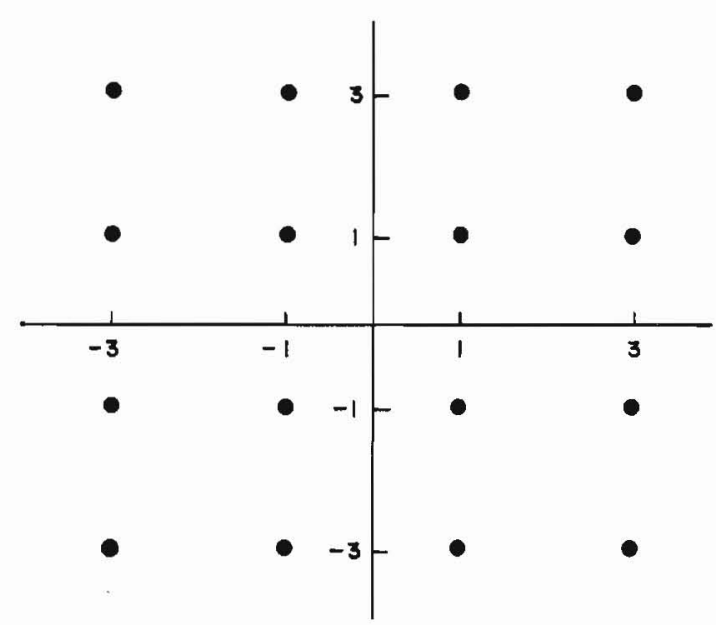

Figura 5. Constelação $Q A M-16$.

$\operatorname{MSE}(d B)$

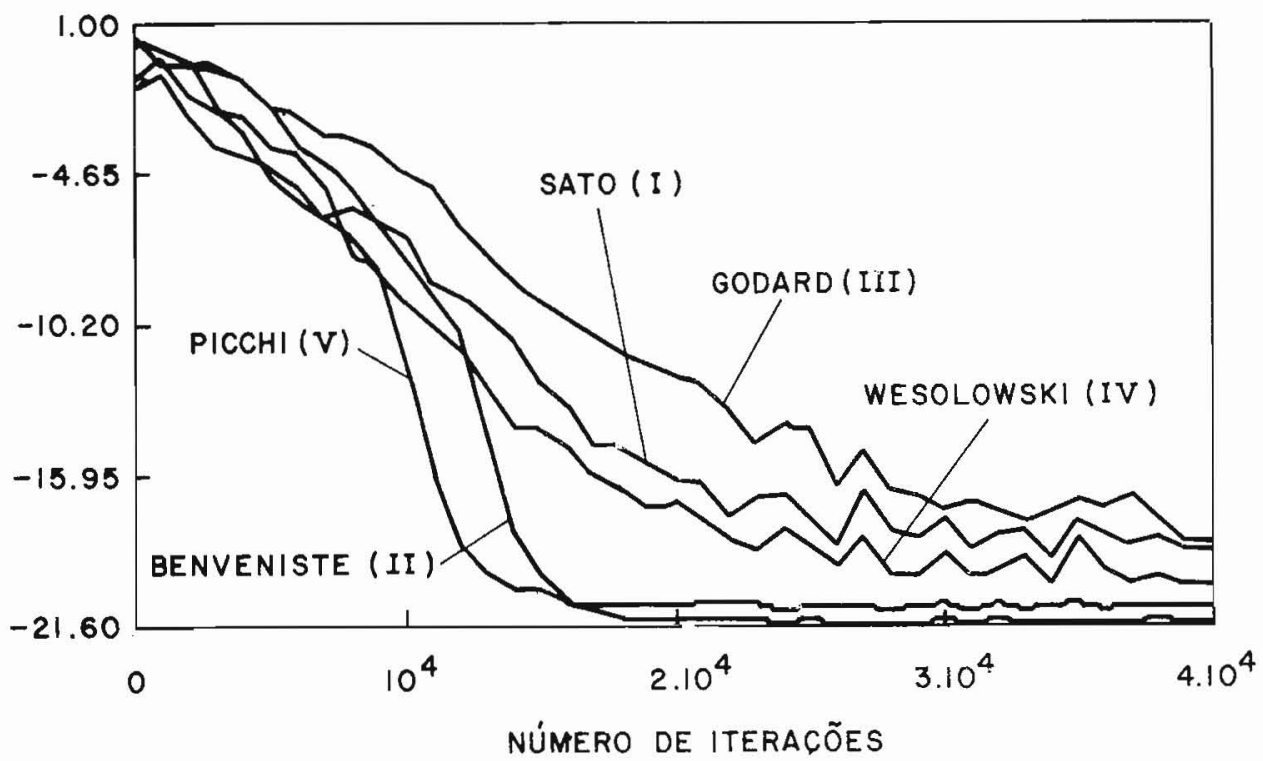

Figura 6. Erro quadrático médio em função do número de iterações quando os algoritmos são utilizados em conexão com o canal 1. 




Figura 7. Erro quadrático médio em função do número de iterações quando os algoritmos são utilizados em conexão com o canal 2.

\begin{tabular}{c|c}
\hline Algoritmo & \multicolumn{1}{c}{ Parâmetros } \\
\hline I & $B=2,5 ; \mu=0,0008$ \\
II & $\beta=2,5 ; \mu=0,0003 ; \mathrm{k}_{1}=4 ; \mathrm{k}_{2}=2$ \\
III & $\mathrm{R}=13,15 ; \mu=0,00008$ \\
IV & $\mathrm{A}=8,2 ; \mu=0,0001$ \\
V & $\beta=2,5 ; \mu=0,004$ \\
\hline
\end{tabular}

Tabela 2. Parâmetros utilizados na implementação dos algoritmos comparados nas figuras 6 e 7.

Várias conclusões podem ser extraídas das figuras.

1) $O$ algoritmo $V$ é o que converge mais rapidamente, pois permite utilizar um valor de $\mu$ mais elevado do que aqueles que podem ser utilizados em conexão com os demais algoritmos.

2) Pelo menos no que se refere ao canal 1, os algoritmos II e $V$ apresentam desempenhos semelhantes, o que está de acordo com as simulações descritas

70 
em [8]. Além disso, no estado estacionário, o comportamento de ambos se aproxima daquele do algoritmo convencional pois, para o algoritmo $\mathrm{II}$, o primeiro termo do segundo membro de (7) prevalece sobre o segundo, e para o algoritmo $V$ as funções $f_{r}(n)$ e $f_{i}(n)$ assumem o valor 1. Esta característica pode ser entendida como uma chave automática que realiza a comutação entre os algoritmos cego e convencional de acordo com o MSE na saída do equalizador.

3) Como esperado, no estado estacionário, os algoritmos I, III e IV apresentam um MSE sensivelmente maior que os algoritmos II e V. Este comportamento pode ser melhorado diminuindo-se gradativamente o valor do passo de adaptação $(\mu)$ à medida que o valor do MSE vai se aproximando do valor mínimo. No presente trabalho, 0 valor de $\mu$ foi mantido constante (no valor que resultou na maior velocidade de convergência), não só para tornar a comparação mais clara, como também por ser este um procedimento comumente adotado na prática.

4) Como o algoritmo convencional, todos os algoritmos descritos são sensíveis ao espalhamento dos autovalores da matriz de autocorrelação do sinal à entrada do equalizador. Em particular, os algoritmos II e V dependem do símbolo decidido â(n) e, portanto, quanto menor for a iis inicial (a qual é diretamente proporcional à razão $\lambda_{\max }\left(\lambda_{\min }\right)$, maior o número de símbolos decididos corretamente e, conseqüentemente, maior a velocidade de convergência.

\section{Conclusões}

Neste trabalho foram examinados e comparados diversos algoritmos de equalização adaptativa que convergem sem a necessidade de uma seqüência de treinamento específica, como tradicionalmente se faz no algoritmo convencional. As simulaçōes realizadas com canais telefônicos típicos mostraram que a convergência ocorre por volta de 10.000 iterações, o que corresponde a aproximadamente 4 segundos para a taxa de 2.400 baud. Como é mostrado em [3], com uma seqüência de treinamento adequada no algoritmo convencional, pode-se atingir a convergência em cerca de 400 iterações mas, como foi comentado no início do trabalho, a utilização destas seqüências nem sempre é uma estratégia eficiente e, nestes casos, os equalizadores cegos são a solução mais adequada, apesar da menor velocidade de convergência. Outro aspecto importante é que a complexidade computacional introduzida nos algoritmos de equalização cega em relação ao algoritmo convencional é pequena, de modo que eles podem ser implementados sem maiores dificuldades.

Os algoritmos descritos aqui são aplicáveis ao equalizador transversal com espaçamento $T$ (inverso da taxa de símbolos) entre as derivações. Recentemente, entretanto, foram propostos algoritmos de equalização cega para equalizadores com espaçamento fracionário (menor que T) [11], e para estruturas em treliça [12], que convergem com velocidade significativamente maior. 


\section{Referências}

[1] S. Haykin, "Adaptive Filter Theory", Prentice-Hall, New York, 1986.

[2] D. N. Godard, "Self-Recovering Equalization and Carrier Tracking in Two-Dimensional Data Communication Systems", IEEE Transactions on Communications, vol. COM-28, n. 11 , Novembro 1980, pp. $1867-1875$.

[3] R. S. Lucatelli, "Equalização Adaptativa para Modems de Dados", Tese de Mestrado, Universidade Estadual de Campinas-UNICAMP, Junho 1988.

[4] B. Widrow e M. E. Hoff, "Adaptive Switching Circuits", IRE WESCOM Conference Record, 1960, pp. 96-104.

[5] Y. Sato, "A Method of Self-Recovery Equalization for Multilevel Amplitude-Modulation Systems", IEEE Transactions on Communications, vol. COM-23, n. 6 , Junho 1975, pp. 679-682.

[6] A. Benveniste e M. Goursat, "Blind Equalizers", IEEE Transactions on Communications, vol. COM-32, n. ${ }^{\circ} 8$, Agosto 1984, pp. 871-883.

[7] K. Wesolowski, "Self-Recovery Adaptive Equalization Algorithms for Digital Radio and Voiceband Data Modems", European Conference on Circuit Theory and Design Proceedings, Paris, França, Setembro 1987, pp. 19-24.

[8] G. Picchi e G. Prati, "Blind Equalization and Carrier Recovery Using a 'Stop-and-Go' Decision-Directed Algorithm", IEEE Transactions on Communications, vol. COM-35, n. ${ }^{\circ}$, Setembro 1987, pp. 877-887.

[9] O. Marcchi e E. Eweda, "Convergence Analysis of Self-Adaptive Equalizers", IEEE Transactions on Information Theory, vol. IT-30, n. 2 , Março 1984, pp. 161-176.

[10] G. J. Foschini, "Equalizing Without Altering or Detecting Data", AT\&T Technical Journal, vol. 61, n. 8 , Outubro 1985, pp. 1885-1911.

[11] G. Picchi e G. Prati, "A Blind SAG-SO-DFD-FS Equalizer", IEEE International Conference on Communications, Philadelphia, Estados Unidos, Junho 1988, pp. 30.4.1-30.4.5.

[12] V. Weerackody e S. A. Kassam, "Blind Equalization Using Lattice Filters", IEEE Internacional Conference on Communications, Philadelphia, Estados Unidos, Junho 1988, pp. 12.5.1-12.5.4. 
ROBERTO SILVA LUCATELLI ARAUJO graduou-se em Engenharia Elétrica pela Universidade de Brasília em 1982. Obteve o Grau de Mestre em Comunicações pela Universidade Estadual de Campinas em 1988. Atualmente trabalha no CPqD - Telebrás no Projeto Rede Digital de Serviços Integrados (RDSI). Suas principais áreas de interesse são Comunicação de Dados e Filtragem Adaptativa.

FÁBIO VIOLARO graduou-se em Engenharia Elétrica em 1973, obteve o grau de Mestre em 1975 e o grau de Doutor em 1980, todos pela Faculdade de Engenharia Elétrica da Universidade Estadual de Campinas (UNICAMP), onde é atualmente Professor. Suas áreas de interesse se concentram em Comunicações Digitais, Equalização Adaptativa e Processamento de Voz. 